\title{
NUMERICAL STUDY OF THE AERODYNAMIC PERFORMANCE OF NACA 0012 IN THE PRESENCE OF AN UNSTEADY HEAT SOURCE
}

\author{
M.A. Mendez Soto, D.P. Porfiriev \\ Samara National Research University, Samara, Russia
}

\begin{abstract}
The objective of this paper is to study the effect of an unsteady moving heat source on the aerodynamic performance of an NACA 0012 airfoil section, with particular focus on the lift and drag coefficients. The compressible Navier-Stokes equations are solved using a finite volume method as well as Spalart-Allmaras Model for turbulence simulation. The heat source periodically moves over the lower surface of the airfoil in the downstream direction. The numerical results show how the drag and lift coefficient strongly depend upon the velocity of the source. For a constant source power, a progressive improvement in the mean values of lift and drag coefficients is observed as velocity increases.
\end{abstract}

Keywords: aerodynamic performance, heat source, lift coefficient, drag coefficient, computational fluid dynamics.

Citation: Mendez Soto MA., Porfiriev DP. Numerical study of the aerodynamic performance of NACA 0012 in the presence of an unsteady heat source. CEUR Workshop Proceedings, 2016; 1638: 498-507. DOI: 10.18287/16130073-2016-1638-498-507

\section{$1 \quad$ Introduction}

Optimizing aerodynamic performance and increasing the reliability of flying machines has led scientists to find new methods that simultaneously provide increase in the lift and reduce drag. One of those approaches has been using procedures based on heat transfer effects.

Over the last decades, several numerical and analytical methods to study heat effects have been developed. Generally, the effect is studied by imposing steady temperature differences between the airfoil surface and the freestream. For example, Norton et al. (1973) [1] studied the case of NACA 0012 heated at different ratios and considering both laminar and turbulent flows over the surface. Their results showed a destabilization of the boundary layer, earlier transition and separation for temperature ratio bigger than unity. The paper showed a reduction in the value of $\mathrm{Cl}_{\max }$ and an increase in drag as the airfoil was heated. 
Similar findings were found by Blohm and Marchman (1974) [2], who studied the heat transfer effects in subsonic flow around a delta wing. In this case, considering the effect on leading edge vortices.

Fleming and Taylor (1992) [3] implemented a computed model of incompressible turbulent flow and heat transfer over rough surfaces introducing it into an existing NavierStokes algorithm. Similarly, Allen and Look [4] created a method to calculate heat transfer rate for wings and bodies of revolution over the surface as a chord wise distribution.

The effects of heat transfer on boundary layer stability and laminar-turbulent transition were pointed out by investigations such as the one carried out by Landrum and Macha (1987) [5], which experimentally presents the effect of heating the nose of a NACA 0012 on turbulence disturbances. Transition location was practically unaffected, but transition zone resulted larger and the amplitude of disturbances increased with heating. A later analytical investigation conducted by Kazakov et al. (1995) [6] showed the possibility of delaying transition in airfoils by heating a tiny area near the leading edge and an improvement in lift generation was achieved by varying the pressure distribution. More effects on shear layer development for attached and separated layers are discussed by Mabey (1990) [7], who also includes analysis of velocity profiles, thickness and transition of the boundary layer. The author points out that heat transfer has powerful effects on aerodynamic measurements that should not be neglected.

Motivated by the appearance of unmanned and micro aerial vehicles, numerous researchers have investigated heat transfer effects on flow with very low Reynolds and Mach numbers. By cooling the extrados and heating the intrados, Kim et al. (2003) [8] achieved to enhance lift and reduce drag, especially significant for small-scale airfoils. The same results were obtained by Bekka et al. (2009) [9] using a numerical computation of the flow around microscale wing for MAV with and without thermal effect. A more specific study of influence of heat transfer on the aerodynamic performance of a plunging and pitching NACA0012 airfoil at low Reynolds numbers can be found in Hinz et al. (2013) [10].

Thus, practically no attention has been drawn to the effects that an unsteady heat source might cause on aerodynamic performance of airfoils. Similarly, most of the investigations focus on the influence produced specifically on the boundary layer. However, it is worth mentioning that there are experimental and theoretical studies that have shown an improvement in lift generation by adding a moving unsteady heat source for the flow around a cylinder [11].

Given the lack of investigation in this area, the objective of the current study is to present the possible effect of an unsteady heat source in airfoil aerodynamics. As a framework for the numerical simulations the 2D NACA 0012 Validation Case of NASA Langley Research Centre is used here [12]. All the numerical calculations of this research were computed on the supercomputer "Sergey Korolyov" at Samara State Aerospace University using the software ANSYS Fluent 15.0. 


\section{Theory and simulation parameters}

\subsection{Governing equations}

The governing equations are two-dimensional unsteady compressible Reynoldsaveraged Navier-Stokes equations (RANS), which in an Einstein notation can be expressed as follows (e.g. Anderson et al (2010) [13] and Bekka (2009) [9]):

$\frac{\partial \rho}{\partial t}+\nabla \cdot\left(\rho u_{i}\right)=0$,

$\frac{\partial\left(\rho u_{i}\right)}{\partial t}+\frac{\partial\left(\rho u_{i} u_{j}\right)}{\partial t}=\frac{\partial \hat{\tau}_{i j}}{\partial x_{j}}-\frac{\partial p}{\partial x_{j}}$,

$\frac{\partial(\rho E)}{\partial t}+\frac{\partial\left(\rho E u_{j}\right)}{\partial x_{j}}=\frac{\partial}{\partial x_{j}}\left(u_{i} \hat{\tau}_{i j}-q_{j}\right)-\frac{\partial\left(\rho u_{j}\right)}{\partial x_{j}}$,

where $\mathrm{E}$ is the total energy, $\hat{i}_{i j}$ is the stress tensor for molecular and Reynolds stresses, and $q_{j}$ is the total heat flux.

The stress tensor is evaluated using Stokes hypothesis and Boussinesq assumption and can be expressed as:

$$
\begin{aligned}
& \hat{\tau}_{i j}=2 \mu\left(S_{i j}-\frac{S_{k k} \delta_{i j}}{3}\right) \tau_{i j}, \\
& \tau_{i j}=2 \mu_{t}\left(S_{i j}-\frac{S_{k k} \delta_{i j}}{3}\right)-2 \frac{\rho k \delta_{i j}}{3} .
\end{aligned}
$$

For simulations in this paper, the turbulent eddy viscosity $\mu_{t}$ is calculated using Spalart-Allmaras turbulence model. This model was designed and optimised for aerospace applications, especially for flows past wings and airfoil. Some of its advantages include: ease of implementation for any type of grid (e.g. structured or unstructured, single-block or multi-block) and computational efficiency, since it only solves for only a single additional variable, which makes it quite stable and less memoryintensive than the other models [14].

This model solves a single transport equation, written as:

$$
\frac{\partial(\rho \tilde{v})}{\partial t}+\frac{\partial\left(\rho \tilde{v} u_{j}\right)}{\partial x_{i}}=G_{v}+\frac{1}{\sigma_{\tilde{v}}}\left(\frac{\partial}{\partial x_{j}}\left\{(\mu+\rho \tilde{v}) \frac{\partial \tilde{v}}{\partial x_{j}}\right\}+C_{b 2} \rho\left(\frac{\partial \tilde{v}}{\partial x_{j}}\right)^{2}\right)-Y_{v}+S,
$$

where $\mu_{t}=\rho \tilde{v} f_{v 1}$. A more detailed description of variables and constants included in this model can be found in Spalart and Allmaras (1994) [15]. 
Unsteady RANS are numerically solved in ANSYS Fluent by using the finite volume method, which rewrites a general scalar transport equation as an algebraic expression that can be calculated for each control volume [16]. The base of the method is the integral form for the transport of a scalar quantity $\phi$ in an arbitrary volume $V$, as follows:

$\int_{V} \frac{\partial \phi}{\partial t} d V+\llbracket \rho \rho \vec{v} \cdot d \vec{A}=\llbracket \int \Gamma_{\phi} \nabla \phi \cdot d \vec{A}+\int_{V} S_{\phi} d V$

where $\rho$ - density, $\vec{v}$ - velocity vector, $\vec{A}-$ surface area vector, $\Gamma_{\phi}-$ diffusion coefficient for $\phi$ and $S_{\phi}$ source of $\phi$ per unit volume. Applying discretization to (7), it yields:

$\frac{\partial \phi}{\partial t} \cdot V+\sum_{i=1}^{N} \rho_{i} \phi_{i} \vec{v}_{i} \cdot \vec{A}_{i}=\sum_{i=1}^{N} \Gamma_{\phi} \nabla \phi_{i} \cdot \vec{A}_{i}+S_{\phi} \cdot V$,

where $N$ is the number of faces enclosing a cell and the values $\frac{\partial \phi}{\partial t}$ of $\phi_{i}$ and are determined using temporal and spatial discretization schemes, respectively.

In the spatial discretization, the Third-Order MUSCL (Monotone Upstream-Centered Schemes for Conservation Laws) scheme was used to evaluate the final solution for density, momentum, turbulent viscosity and energy. Similarly, Green-Gauss nodebased and second-order upwind schemes are applied for gradient and pressure evaluation, respectively. In the temporal discretization, a second-order implicit method is assigned.

Additional models, to solve the closure problem of unsteady RANS equations, include ideal-gas law, constant specific heat assumption and Sutherland's model for dynamic viscosity.

\subsection{Domain and boundary conditions}

An existing structured $1793 \times 513$ grid is recompiled from [12] and used as computational domain. The grids have a farfield extent of about 500 chord lengths and no farfield point vortex boundary condition correction is being applied in this investigation. The flow domain is scaled twice in order to achieve chord Reynolds Number equal to 6 million.

The same boundary conditions are imposed, as in the case of NASA validation case. These conditions include free stream Mach number of 0.15, chord Reynolds number equal to 6 Million and the angle of attack equal to 10 degrees. For Sutherland's Model the reference viscosity and temperature are $1.865 \cdot 10-5 \mathrm{~kg} / \mathrm{ms} 300 \mathrm{~K}$, respectively and at the far stream boundary the turbulent initial value is set using an initial turbulent viscosity ratio equal to 0.210438 . The airfoil surface is modeled as a wall with no slip and adiabatic conditions. For the Spalart-Allmaras Model a Prandtl Number of 0.72 is used, as well as a value of 0.9 for Energy and Wall Prandtl Numbers, respectively. 
A schematic of the modified flow domain and boundary conditions is presented in Fig. 1.

The unsteady heat source has a circular shape with a 0.005 -meter radius. Timedependent movement is set to be periodic and uniform along the lower surface in the downstream direction. The power of source does not vary over time and its value is constant within the area of the source. Fig. 2 depicts the contours of static temperature in the case of a source with velocity of $30 \mathrm{~m} / \mathrm{s}$.

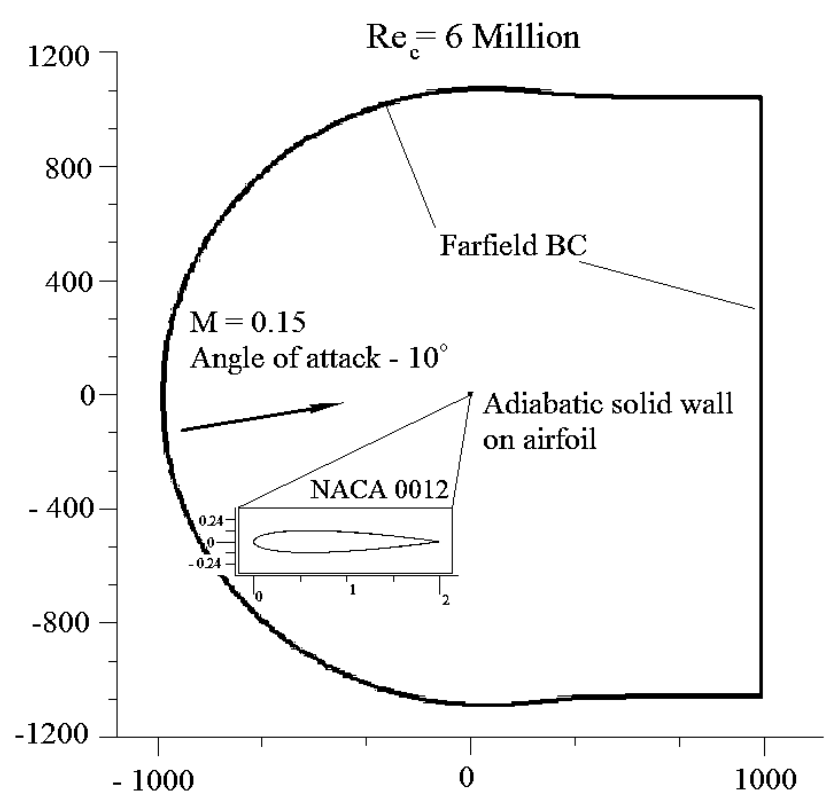

Fig. 1. Schematic of the modified computational domain and boundary conditions

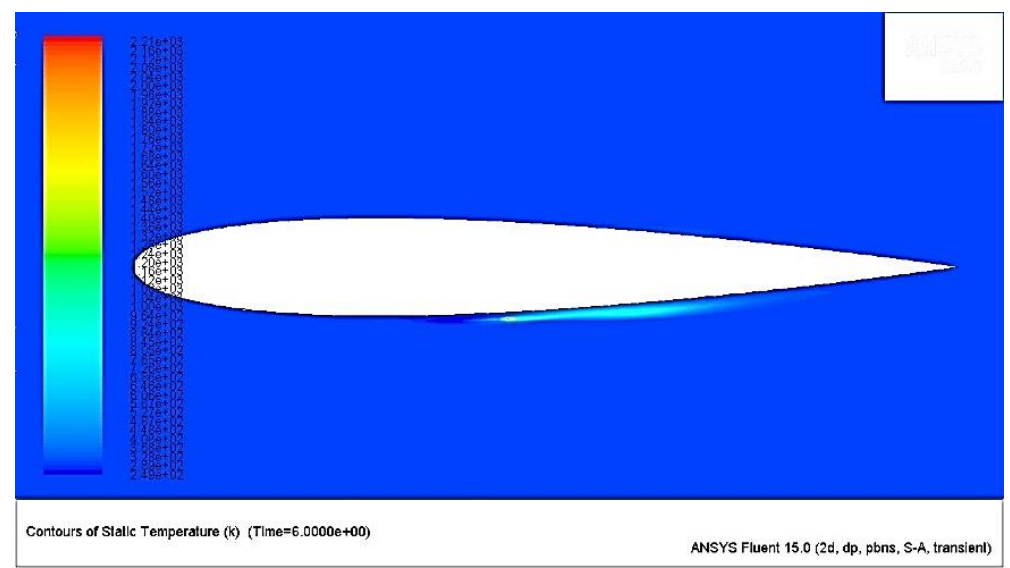

Fig. 2. Contours of static temperature in the case of a source with velocity of $30 \mathrm{~m} / \mathrm{s}$ 


\section{Results and discussion}

\subsection{Grid validation study}

In order to determine the results sensitivity to grid refinement, three different grids have been employed and tested for the case of no source present in the flow. $C_{D}$ and $\mathrm{C}_{\mathrm{L}}$ results are further compared with the solutions available in the open source for the Validation and Verification of Turbulence Modelling of NASA Langley Center [12]. Their numerical study obtained values of 1.09094 and 0.01227275 for $C_{L}$ and $C_{D}$, respectively.

The details of the three grids are given in Table 1.

Table 1. Description of grids over NACA0012 airfoil used for grid independence study

\begin{tabular}{ccc}
\hline Grid & $\begin{array}{c}\text { Total Number of } \\
\text { Cells }\end{array}$ & $\begin{array}{c}\text { Points on the airfoil sur- } \\
\text { face }\end{array}$ \\
\hline Grid 1 & 3729 & 65 \\
Grid 2 & 57921 & 257 \\
Grid 3 & 919809 & 1025 \\
\hline
\end{tabular}

The values obtained for $C_{D}$ and $C_{L}$ for the three grids, as well as the corresponding errors are presented in Table 2. As the number of cells increase, the value for $C_{D}$ and $\mathrm{C}_{\mathrm{L}}$ decreases and asymptotically approaches the values numerically obtained and validated in [12]. For the purpose of numerical investigations and considering the available computational capacity, a grid of at least 919809 cells is selected, which provides errors of less than $0.45 \%$ for $\mathrm{CD}$ and $0.035 \%$ for CL. Thus, the results obtained using Grid 3 are further used as reference values for comparisons in the following sections of this paper.

Table 2. Comparison of $\mathrm{C}_{\mathrm{D}}$ and $\mathrm{C}_{\mathrm{L}}$ obtained using different grids

\begin{tabular}{ccccc}
\hline Grid & $\mathbf{C}_{\mathbf{D}}$ Value & Error (\%) & $\mathbf{C}_{\mathbf{L}}$ Value & Error (\%) \\
\hline Grid 1 & 0.0157969 & 28.70 & 1.10224 & 1.04 \\
Grid 2 & 0.0123895 & 0.95 & 1.09496 & 0.37 \\
Grid 3 & 0.0123218 & 0.40 & 1.09064 & 0.03 \\
\hline
\end{tabular}

The convergence of $C_{D}$ and $C_{L}$ as the number of cells increases is plotted in Fig. 3.

\subsection{Thermal Effect Study}

To investigate the effect of an unsteady heat source around a NACA 0012, several possibilities for source movement were analyzed. Preliminarily, uniform clockwise movement around the entire surface and movement along only one of the surfaces were considered. The results showed a relative decrease in $C_{L}$ and increase in $C_{D}$ for different velocities. For instance, an application of a $15 \mathrm{~kW}$ source with a uniform 
velocity of $15 \mathrm{~m} / \mathrm{s}$ caused a relative reduction of $11 \%$ in the lift-to-drag, which derived from a $9 \%$ increment in $C_{D}$ and a reduction of $4 \%$ in $C_{L}$. In the case of $100 \mathrm{~m} / \mathrm{s}$ as source velocity, the results did not change much with an $8 \%$ of $\mathrm{C}_{\mathrm{L}}$ decrease and a $2 \% \mathrm{C}_{\mathrm{D}}$ increase.

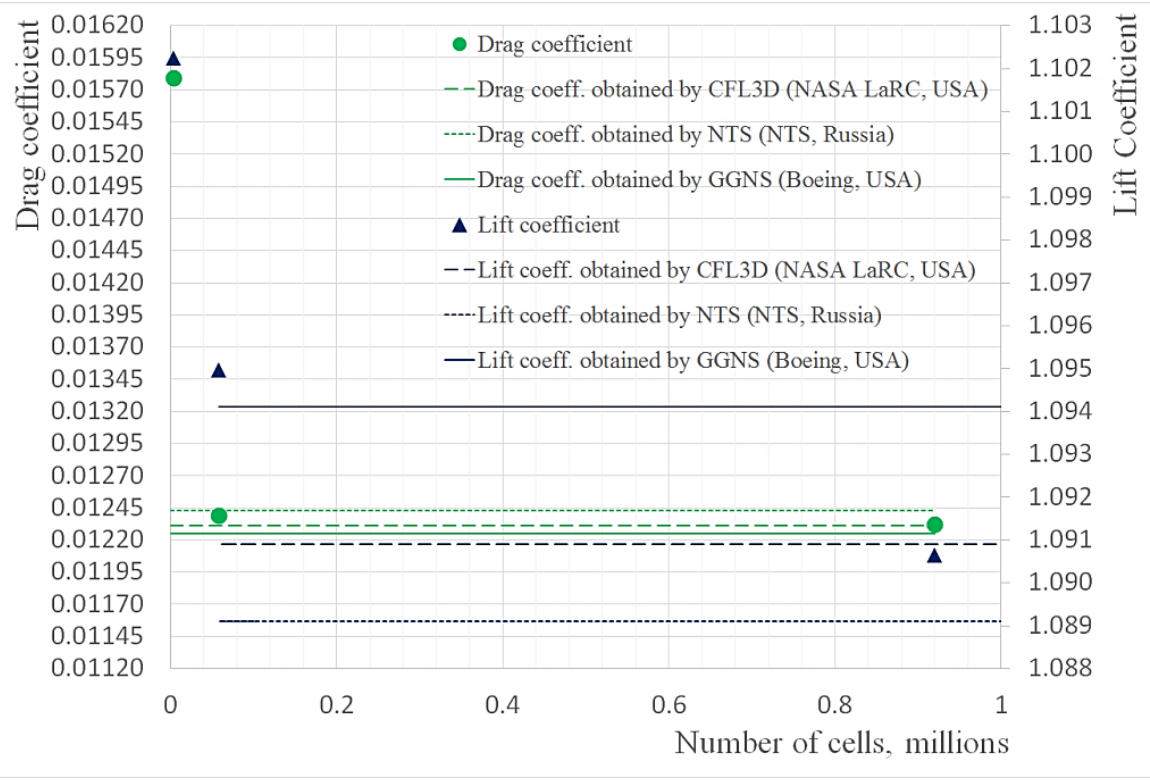

Fig. 3. Convergence of $C_{D}$ and $C_{L}$ for different grid refinements

A posterior analysis of the overall pattern of the data showed a slight improvement in the aerodynamic characteristics as the source moved along the lower surface. Thus, a source movement only along the lower surface was chosen as focus of investigation. To see the effect of source velocity on the aerodynamic performance of NACA 0012 , four simulations are conducted for different velocities using a constant source power value of $30 \mathrm{~kW}$. Fig. $4 a$, b shows the change in $C_{D}$ and $C_{L}$ mean values in the presence of the unsteady heat source with different velocities.

The data demonstrates a monotonic decrease of $C_{D}$ as velocity becomes higher, whereas $\mathrm{C}_{\mathrm{L}}$ monotonically increase for increasing source velocity. Besides, gradient of increment in $\mathrm{C}_{\mathrm{L}}$ seems to gradually diminish with increasing source velocity.

Despite a relative increment of $C_{D}$ mean values with respect to $C_{D}$ obtained with no source present, the overall aerodynamic performance tends to increase since $C_{L}$ also reaches higher values. Nevertheless, this overall improvement is only comparatively better for high velocities. These results suggests the possible existence of a minimum source velocity necessary to observe an enhanced aerodynamic performance for a specific source power. The determination of this minimum velocity is beyond the scope of this article. 

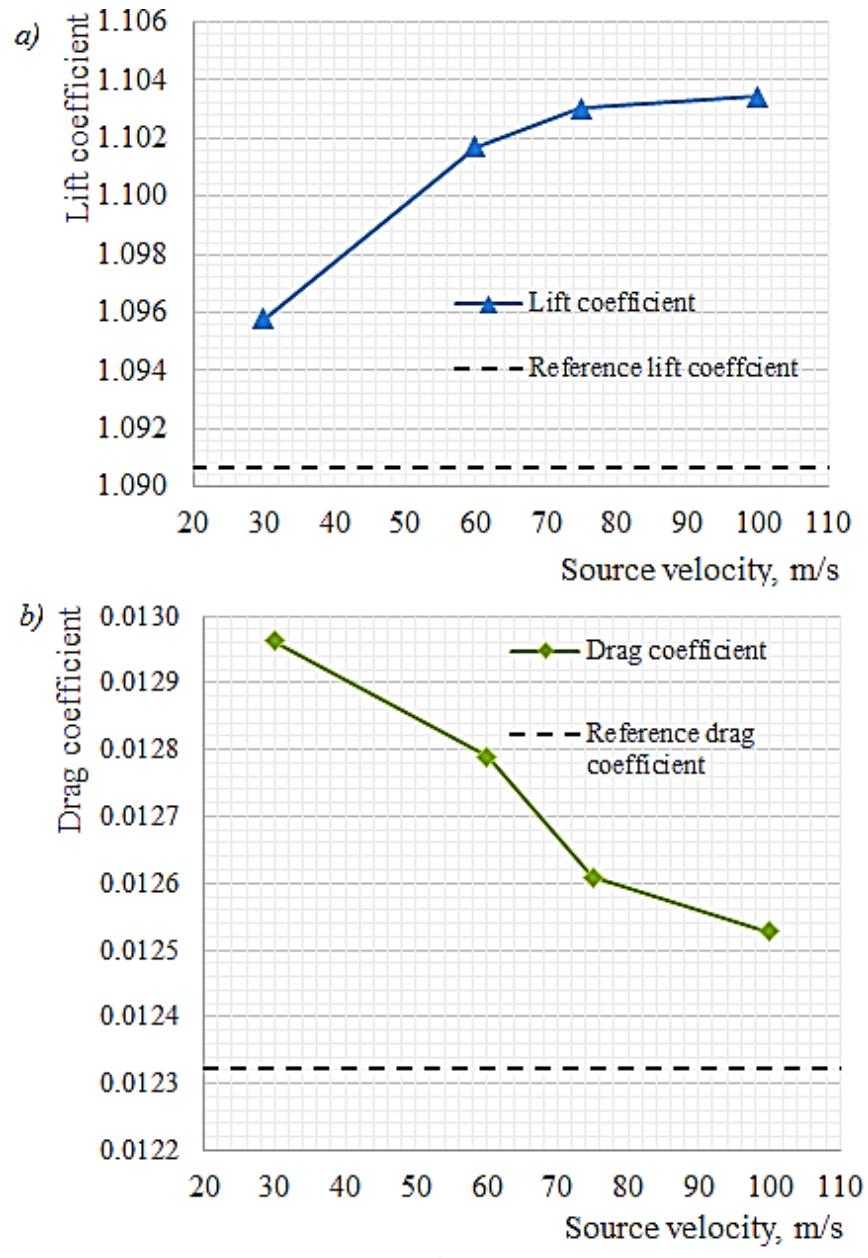

Fig. 4. Mean value of lift (a) and drag (b) coefficients for different source velocities

\section{Conclusions}

The effect of an unsteady moving heat source on the aerodynamic performance of NACA 0012 was investigated using numerical simulation. Computations are performed varying velocity source and using a constant power. In all cases, the computed results are performed using second order implicit scheme for time integration and third order MUSCL and second-order upwind schemes for spatial integration. Preliminarily, an investigation into the sensitivity of the results to grid refinement is conducted; furthermore, the results are verified based on solutions available in [12]. Simulation results reveal that velocity increment has positive effects on aerodynamic performance both increasing lift coefficient and decreasing drag coefficient. 
In future papers, more attention has to be paid to the optimization of the source parameters in order to obtain a lift-maximized aerodynamic performance. A more detailed range of values for velocity and power could be considered as well as a further frequency study of the oscillations for $C_{D}$ and $C_{L}$ coefficients.

\section{Acknowledgments}

The study was supported in part by the Ministry of Education and Science of the Russian Federation under the Competitiveness Enhancement Program of SSAU (20132020) and by State Assignment to Educational and Research Institutions under Project Nos. 102, 608.

\section{References}

1. Norton DJ, Macha JM, Young JC. Surface temperature effect on subsonic stall. Journal of Spacecraft and Rockets, 1973; 10: 581-587.

2. Blohm RW, Marchman JF. Heat transfer effects on a delta wing in subsonic flow. In: Proceedings of the 1974 Heat Transfer and Fluid Mechanics Institute, 1974: 220-235.

3. Fleming KR, Taylor RP. Incompressible Navier-Stokes Algorithm for flow and Heat transfer over rough surfaces, AIAA 27th thermophysics conference, July-6-8, AIAA, 1992; 2925.

4. Allen J, Look BC. A method for calculating heat transfer in the Laminar flow region of bodies, NACA, Report no. 764: 178-182.

5. Landrum DB, Macha JM. Influence of a heated leading edge on boundary layer growth, stability and transition. In: Proceedings of the 19th Fluid Dynamics, Plasma Dynamics and Laser Conference, Honolulu, Hawaii, 1987.

6. Kazakov AV, Kogan MN, Kuparev VA. Optimization of laminar-turbulent transition delay by means of local heating of the surface. Fluid Dynamics, 1995; 30: 563-570.

7. Mabey DG. Effects of heat transfer on aerodynamics and possible implications for wind tunnel tests. Progress in Aerospace Sciences, 1990; 27: 267-303.

8. Kim J, Rusak Z, Koratkar N. Small-scale airfoil aerodynamic efficiency improvement by surface temperature and heat transfer. AIAA Journal, 2003; 41: 2105-2113.

9. Bekka N, Bessaih R, Sellam M. Numerical study of heat transfer around the smallscale airfoil using various turbulence models. Numerical Heat Transfer Part A: Applications, 2009; 56: 946-969.

10. Hinz DF, Alighanbari H, Breitsamter C. Influence of heat transfer on the aerodynamic performance of a plunging and pitching NACA0012 airfoil at low Reynolds numbers. Journal of Fluids and Structures, 2013; 37: 88-99.

11. Bitjurin BA, Zavershinskij IP, Klimov AI, Molevich NE, Moralev IA, Munhoz D, Poljakov LA, Porfiriev DP, Sugak SS. Flow around a cylinder in the presence of 
an electric arc, rotating in a magnetic field. High Temperature, 2016; 54(4). [In Russian]

12. 2D NACA 0012 Airfoil Validation Cases available atю URL: http://turbmodels. larc.nasa.gov/naca0012numerics_val.html .

13. Anderson JD, Gerard D, Dick E. Computational Fluid Dynamics: An Introduction. Ed. John F. Wendt. Third ed. Berlin: Springer, 2010.

14. Argyropoulos C, Markatos N. Recent advances on the numerical modelling of turbulent flows, Applied Mathematical Modelling, 2012; 39: 693-732.

15. Spalart P, Allmaras S. A One-Equation Turbulence Model for Aerodynamic Flows. Recherche Aerospatiale. American Institute of Aeronautics and Astronautics, 1994; 5-21.

16. ANSYS, Inc. ANSYS Fluent Theory Guide. ANSYS, Inc., PA, USA, 2013. 